absorption in thorium was studied in relation to conversion factor, that is, breeding uranium-233 fuel from thorium. The technical physics section provided additional safety instrumentation for Hifar and designed and constructed a wide variety of instruments for nucleonic and physical measurements. Generally, more emphasis was placed on the hightemperature gas-cooled reactor project than on the liquid metal cooled reactor system since it was shown that this system would require a longer period of development. Work on liquid metals was largely restricted to sodium technology required for Hifar irradiations. Optimum conditions for the vacuum hot-pressing of reduced beryllium powder and electro. lytic powder were established, and laboratory-scale extrusion processes for beryllium metal were developed. Research on the impregnation of graphite with furfuryl alcohol to produce graphite of very low gas permeability was carried out, and a number of sample fuel elements of uranium and thorium dispersed in a diluent were prepared.

The number of staff at the Establishment rose from 295 to 484 during the year, but there was difficulty in recruiting research officers of the required ability and experimental officers. The Commission was represented at the inaugural meeting of the council of the Australian Institute of Nuclear Science and Engineering which was held on December 4, 1958, and at the Second International Conference on the Peaceful Uses of Atomic Energy in Geneva. The major publication during the year under review was "Australian Atomic Energy Symposium, 1958", the record of the proceedings of the symposium on the Peaceful Uses of Atomic Energy in Australia, held in Sydney during June 1958. Other publications included the booklet entitled "An Introduction to Nuclear Science", prepared at the request of the New South Wales Department of Education as a teaching aid in schools, and the quarterly journal Atomic Energy. A total of approximately $£ A .30,000$ was provided for extramural research contracts to universities. Thirty-five separate research programmes were being undertaken in eight universities and two hospitals and except for a few which were held up by staffing difficulties they were making good progress. Several were nearing completion. No new undergraduate scholarships were awarded, but fifteen undergraduate students continued their training in addition to forty postgraduate research studentship holders.

\title{
PUBLIC OPINION POLLS IN JAPAN
}

$\mathrm{P}^{\mathrm{v}}$ UBLIC opinion polls are an item of American culture which the Japanese have adopted since Hiroshima. "At the height of their popularity during 1946-50 polling agencies in Japan conducted surveys on everything from attitudes towards the Emperor, housing and daylight saving time, to telephone service, prostitution, school lunches and Typhoon "Jane,". Dr. Matsumoto, who is chief of the Department of Medical Sociology of the Atomic Bomb Casualty Commission, is very well aware of the limitations of this kind of democratized publicity, but he has had the interesting idea of investigating how far this widely ranging material displays a consistent pattern which is in contrast to the ethos of traditional Japanese society*. He considers four main fields of opinion : attitudes towards family and sex relationships, attitudes towards occupation and labour, attitudes towards village life, attitudes towards political leadership.

* Contemporary Japan : the Individual and the Group. By Yoshiharu Scott Matsumoto. Transactions of the American Philosophical Society. New Series, Vol. 50, Part 1. Pp.
Philosophical Society, 1960.) 2 dollars.
After a brief description of the orthodox values of the traditional society, Dr. Matsumoto gives the results of a large number of opinion surveys dealing with such questions as: Should an individual choose his own marriage partner or should the parents decide? Do you feel that abortion should generally be permitted? Is there a social necessity for organized prostitution? Do you think the labour unions are protecting the interests of the workers? How do you feel about the abdication of the present Emperor? The book ends with an "Interpretation and Summary" which is sensible but not profound. It scarcely needed a study of this scale to discover that "Japan has been under strong pressure to conform to Western ideology in her post-war situa. tion. On one hand individualistic orientations are heralded; on the other communistic values are proclaimed". There is a good deal of such writing, but so long as Dr. Matsumoto evades the pressure to conform to the clichés of American sociology he is quite fascinating. The bibliography of Japanese sociological studies at the end of the book should be very useful to specialists.
EDMUND LEACH

\section{MASS DENSITY NEAR THE SUN}

$I^{T}$ $\mathrm{T}$ has been known for many years that there is a close relation between the gravitational force of our Galaxy near, and perpendicular to, its plane, the distribution of stellar velocities and the density distribution of matter. The classical investigation of this subject was made by J. H. Oort in 1932 , and a number of other investigations have been made since that time.

E. R. Hill has recently published (Bull. Astron. Inst. Netherlands, 15, No. 494, 1 ; 1960) a comprehensive discussion and a new analysis of the available data. His work is based on stars of type $K$; this type was chosen because $K$-type stars occur in relatively large numbers at high galactic latitudes and statistical data on their brightness and motions are much more complete than for other kinds of stars. The velocity distribution of the stars perpendicular to the galactic plane has been represented as the sum of three components, two of low velocity and one of high velocity. The distribution of velocities parallel to the plane was studied as a function of height above the galactic plane. The distribution of giant $K$-stars was derived from the observed dis. tribution of all stars of type $K$ by using the observed 